\title{
OS DESAFIOS DA MUDANÇA PARA O ENSINO REMOTO EMERGENCIAL NA GRADUAÇÃO NA UNICAMP - BRASIL
}

\author{
THE CHALLENGES ON MOVING TO EMERGENCY REMOTE EDUCATION FOR \\ UNDERGRADUATE PROGRAMS AT UNICAMP-BRAZIL
}

\begin{abstract}
Eliana Amaral ${ }^{1}$
Soely Polydoro ${ }^{2}$

Resumo: Este artigo relata a resposta da Unicamp à pandemia Covid-19 no âmbito da Graduação. Com a suspensão das atividades presenciais e a adoção do ensino remoto emergencial em março de 2020, cada unidade acadêmica propôs seu plano de ação, mantendo disciplinas remotas total ou parcialmente. Entre as diferentes ações adotadas, destacaram-se apoio ao acesso digital, à permanência, intensificação da formação docente para uso de meios digitais e flexibilização das normativas institucionais, em acordo às deliberações estaduais. Essas mudanças exigiram intenso envolvimento de gestores acadêmicos, professores, discentes e funcionários. Houve uma avaliação qualitativa de relatórios intermediários por curso, quanto às soluções e às preocupações ou dificuldades, nas dimensões logísticas e tecnológicas, pedagógicas e socioafetivas. Entre as lições aprendidas incluem-se maior habilidade de todos para uso de recursos digitais e adoção de estratégias centradas no estudante, com flexibilidade frente ao inesperado. Há ainda muito para enfrentar diante do impacto da pandemia na comunidade universitária.
\end{abstract}

Palavras-chave: Ensino superior; educação digital; pandemia.

\begin{abstract}
This article reports on Unicamp's response to the Covid-19 pandemic in the context of Undergraduate Programs. With the cessation of face-to-face activities and the adoption of emergency remote education in March 2020, each academic unit proposed its action plan, maintaining remote disciplines, total or partially. Among the different actions adopted, support for digital access, for permanence, intensification of teacher training for the use of digital resources and flexibility of institutional regulations in accordance with state deliberations stood out. These changes required intense engagement from academic managers, teachers, students and staff. There was a qualitative evaluation from intermediate reports per course regarding solutions and concerns / difficulties, in the logistical and technological, pedagogical and socio-affective dimensions. Lessons learned include greater ability for everyone to use digital resources and greater adoption of student-centered strategies, with flexibility in the face of the unexpected. We still have a lot to face in view of the impact of the pandemic on the university community.
\end{abstract}

Keywords: Higher education; digital education; pandemia.

\section{Introdução}

O impacto direto da pandemia de Covid-19, que se iniciou na Ásia, passou pela Europa, Oceania, África e chegou às Américas, foi sentido na Universidade Estadual de Campinas (Unicamp) no dia 13 de março de 2020, quando, por deliberação da Reitoria (GR n 24/2020), decidiu-se pela suspensão das atividades presenciais e mudança para atividades de ensino remoto emergencial, mediado por tecnologia digital. Essa suspensão ocorreu uma semana após o início das aulas, trazendo uma situação especialmente desafiadora para a turma de ingressantes 2020. Decidiu-se por postergar

\footnotetext{
${ }^{1}$ Universidade Estadual de Campinas, Campinas, SP, Brasil.

${ }^{2}$ Universidade Estadual de Campinas, Campinas, SP, Brasil.
} 
o semestre letivo até o final de agosto, com a expectativa de que seria possível sua finalização com atividades presenciais. Na evolução do semestre, tornou-se evidente o descontrole da pandemia no estado e na região, com interiorização dos casos e superlotação dos hospitais e leitos para casos graves. Seguindo orientações sanitárias, a Unicamp decidiu, ao final de junho, prolongar a suspensão das atividades presenciais por prazo indeterminado, dependente do controle da pandemia (GR $\mathrm{n}^{\circ}$ 72/2020). Desta forma, o primeiro semestre letivo terá seu encerramento sem atividades presenciais.

Estabeleceu-se um prazo de 30 dias para que as 24 unidades acadêmicas (faculdades ou institutos) preparassem seus planos de ensino emergenciais para o semestre. Ao final, apenas 2,6\% das disciplinas dos 69 cursos de graduação foram canceladas pelas Unidades $(2,2 \%$ obrigatórias e 3,7\% de eletivas). Houve grande mobilização dos docentes, dos discentes e dos gestores acadêmicos nas várias etapas de replanejamento dos cursos de graduação; reorganização das disciplinas; novas estratégias educacionais e tecnologias desconhecidas. Também foi necessária a adaptação de normas acadêmicas. Ao longo do semestre, foram aprovadas deliberações nas câmaras pertinentes, alinhadas às normativas do Conselho Estadual de Educação, órgão regulatório para a Unicamp (Deliberação CEE 177/2020, Parecer 109/2020, Deliberação CEE 185/2020). As mudanças necessárias exigiram flexibilidade administrativa, acadêmica e muita colaboração da comunidade, composta de aproximadamente 2 mil professores, 7,5 mil servidores não docentes e quase 20 mil estudantes de graduação.

Embora a suspensão das atividades presenciais tenha ocorrido em todas as partes do mundo, não há uma orientação prescritiva e única diante de um desafio tão complexo. O estudo de Crawford et al. (2020) sobre a agilidade de ações do ensino superior de 20 países diante da Covid19 demonstra que as universidades e os governos adotaram diferentes respostas. No entanto, sabese que as situações de crise demandam soluções criativas, inovadoras. Assim, Hodges et al. (2020) cunharam o termo "ensino remoto emergencial" (ERE) para representar as circunstâncias excepcionais do processo de ensino-aprendizagem na pandemia. São soluções temporárias, totalmente remotas, adaptadas aos recursos e às possibilidades disponíveis, com manutenção da interação síncrona para mediação, para um ensino que não se caracteriza pela simples transposição das aulas presenciais para o ambiente digital. Difere da educação a distância, online, apoiada num desenho instrucional predefinido, com planejamento cuidadoso por equipes especializadas que desenvolvem um ambiente virtual imersivo para uma experiência educacional essencialmente assíncrona (HODGES et al., 2020; O'KEEFE et al., 2020).

Entre as regulamentações acadêmicas modificadas para mitigar os prejuízos aos estudantes, o prazo de integralização dos cursos foi ampliado em dois semestres e se estendeu o período de cancelamento de disciplinas ou de trancamento até 45 dias antes do encerramento do semestre letivo. Também foi adotada a frequência integral durante o período da suspensão de atividades presenciais. Houve estímulo para criação de disciplinas eletivas interdisciplinares com temáticas voltadas à pandemia, que pudessem ser opção como disciplina exclusiva ou complementar no semestre. Foram modificados os critérios de avaliação, permitindo a opção de avaliação por conceito (suficiente/insuficiente ou falta de informação, a ser retificado posteriormente), com possibilidade de postergar até o término da integralização do curso a conclusão dos componentes de disciplinas que não pudessem ser oferecidos de modo remoto. Também foi facultado ao estudante solicitar exclusão da nota e/ou conceito no cálculo do seu Coeficiente de Rendimento, mesmo que aprovado. Uma medida específica para os ingressantes foi permitir a desistência de matrícula em disciplina e o trancamento do primeiro semestre.

Se as iniciativas de natureza normativa são necessárias, foram essenciais as ações voltadas para a busca de equidade e apoio à permanência dos estudantes. A Unicamp implantou um conjunto de ações para aumentar a inclusão na educação superior, com uso de bonificação para escola pública, cotas raciais, vestibular indígena e vagas olímpicas, o que tem trazido um perfil 
cada vez mais heterogêneo à comunidade acadêmica. Sabe-se que ações de inclusão devem vir acompanhadas por efetivas políticas de permanência, a fim de propiciar o sucesso acadêmico dos estudantes (HERINGER, 2018), o que se intensifica neste momento de crise. Assim, bolsas de apoio à permanência, oferecidas com recursos do orçamento da universidade e gerenciadas pelo Serviço de Apoio ao Estudante - SAE (https://www.sae.unicamp.br/portal/pt/), foram transformadas para o momento de pandemia, e suas atividades associadas adaptadas ao trabalho remoto. $\mathrm{O}$ benefício de auxílio transporte transformou-se em Benefício Emergencial de Atividades Não Presenciais (BENP).

Para o acesso aos equipamentos, foi criado um grupo de voluntários com a participação de professores, funcionários e estudantes sob coordenação do Observatório de Direitos Humanos, instância da Diretoria Executiva de Direitos Humanos (DEDH) (http://www.direitoshumanos.unicamp.br/), que atuou em parceria com a Pró-Reitoria de Graduação (PRG) (https://www.prg.unicamp.br/). Este grupo preparou desktops, notebooks $e$ tablets que, até o momento, foram emprestados para cerca de 700 estudantes. Parte destes equipamentos do acervo da Unicamp estavam sem uso, devido à suspensão das atividades presenciais. Mas também foram adquiridos 340 tablets, 40 notebooks e 500 chips para ampliar o acesso digital. Todo esse processo é realizado com base num cadastro e na assinatura de termo de responsabilidade pelo discente.

Entre os tantos desafios, destaca-se o papel primordial dos professores e a responsabilidade institucional de apoiá-los. Foi necessário dar respostas às especificidades do planejamento de ensino-aprendizagem remoto, facilitar o desenvolvimento de competências digitais, contribuir com o domínio de ferramentas e recursos tecnológicos educacionais, viabilizar novas formas de avaliação, de mediação e facilitação da aprendizagem em ambiente digital e apoiar a reorganização das aulas práticas e de laboratório. Havia familiaridade com o uso dos ambientes virtuais de aprendizagem (AVA) na instituição, mas essa não era uma política instrucional. Da mesma forma, não havia experiência com os recursos de teleconferência para uso rotineiro pelos docentes, e ela era reduzida ao uso de recursos para gravação e disponibilização de aulas (incluindo vídeos e podcasts). Portanto, era primordial apoiar o desenvolvimento de competências digitais pelo corpo docente (REDECKER, 2017).

O aprimoramento da qualidade do ensino na universidade é objetivo do Espaço de Apoio ao Ensino e Aprendizagem $(\mathrm{EA})^{2}$, um órgão da PRG criado em março de 2010 (https://www.ea2.unicamp.br/), que desde então vem promovendo ações de apoio às coordenações de curso, experiências formativas para o desenvolvimento profissional docente e inovações curriculares. Já estavam em andamento vários projetos para estimular a reflexão e a discussão sobre o projeto pedagógico de curso, o planejamento de ensino, as ações pedagógicas e a mediação, o processo de avaliação educacional, as novas ferramentas e estratégias de ensinoaprendizagem, visando à metodologia ativa e à aprendizagem centrada no estudante. Ao mesmo tempo, já vinham sendo oferecidas, pelo Grupo Gestor de Tecnologias Educacionais (GGTE) (https://ggte.unicamp.br/wp/), oportunidades formativas para produção de vídeos, apoio à produção de podcasts, além de cursos para uso das plataformas eletrônicas educacionais e estímulo por editais para a adoção de ferramentas digitais complementares.

Ainda que houvesse essas condições prévias, em face da complexidade do desafio imposto pela Covid-19 à Universidade, foram intensificadas as ações de apoio aos professores, o que já estava indicado na normativa que suspendeu as atividades presenciais. De fato, sabese que o apoio ao professor tem uma ampla gama de efeitos diretos e mediados nas motivações dos estudantes para o ensino remoto (FRYER; BOVEE, 2016). Com esse foco, a parceria entre a PRG, o (EA) ${ }^{2}$ e o GGTE possibilitou que, de forma imediata e em constante atualização, fosse disponibilizada uma página eletrônica como suporte para o ensino digital 
(https://www.ea2.unicamp.br/ensino-digital-2/), com orientações sobre planejamento das disciplinas, organização das aulas e avaliação; tutoriais sobre os AVAs e os aplicativos; vídeos; curadoria com links para banco de aulas, laboratórios remotos e simulações; gravações de webinários; resoluções pertinentes; artigos e materiais externos de orientação; e acesso ao atendimento via chat e e-mail. Entre março e julho, o site do (EA) ${ }^{2}$ teve pouco mais de 66 mil visualizações, com pico diário em 17 de março (2.245) e mensal em junho (16 mil).

De forma complementar, tiveram início, no final de abril, webinários para troca de experiências com universidades internacionais e nacionais sobre a transição e o desenvolvimento do ensino remoto emergencial, incluindo os tópicos como docência, desafios ao ensinar e ao aprender, interatividade, portfólio cooperativo, avaliação, laboratório remoto, licenciatura. Esses eventos contaram com aproximadamente 1.700 participantes, sendo em torno de $45 \%$ da comunidade Unicamp e os demais vinculados predominantemente a instituições federais de ensino superior das diferentes regiões do Brasil, mas também a instituições privadas, outros órgãos governamentais e associações.

Quanto às plataformas educacionais, a Unicamp criou e utilizava seu próprio AVA desde 1997 (Teleduc), que vinha sendo progressivamente substituído pelas plataformas Moodle ${ }^{\circledR}$ e, posteriormente, com a opção também pelo Google Classroom®. Esses ambientes são administrados pelo GGTE. Os dados mostram que, no primeiro semestre de 2020, estavam inscritos 53 mil usuários nesses AVAs, sendo 1617 docentes no Moodle ${ }^{\circledR}$ e 1005 no Google Classroom ${ }^{\circledR}$. Nas disciplinas/turmas de graduação, foi registrado um crescimento do uso em $31,76 \%$, comparativamente aos valores observados no primeiro semestre de 2019. Também já eram disponíveis recursos adicionais do Google Apps for Education ${ }^{\circledR}$ e Microsoft Office 365 Education ${ }^{\circledR}$ (https://www.ccuec.unicamp.br/).

De acordo com o Observatório de Inovação Educativa da Universidad Tecnológica de Monterrey, envolvendo mais de 800 professores universitários da América Latina, 3 em cada 4 docentes não se percebiam preparados para incorporar ferramentas digitais exigidas no ensino remoto (ARIAS et al., 2020). Como observam Dias-Trindade, Moreira e Ferreira (2020), as plataformas educacionais são frequentemente usadas para repositório de conteúdo, explorando-se pouco seus recursos de atividades e interação, que devem caracterizar o ensino remoto. Além disso, o vasto menu de recursos e ferramentais educacionais digitais disponíveis gera dificuldade de tomada de decisões pelo professor. Assim, há a necessidade do domínio não só sobre os recursos, mas sobre como melhor utilizá-los, fomentando o verdadeiro engajamento dos estudantes elemento essencial neste momento de ensino remoto em meio à pandemia. Dada a escala de migração para a aprendizagem digital e a situação de estresse associada, é fundamental oferecer suporte técnico, pedagógico e psicológico aos docentes (FLORES; GAGO, 2020; HODGES et al., 2020). Na Unicamp, a partir de julho, o Serviço de Apoio Psicológico e Psiquiátrico (SAPPE) (https://www.prg.unicamp.br/graduacao/sappe-2/) e o Centro de Saúde da Comunidade (CECOM) (https://www.cecom.unicamp.br/) têm oferecido apoio psicológico e psiquiátrico aos docentes. Nesta adaptação emergencial, os coordenadores de disciplinas também contaram com a inestimável ajuda de bolsistas graduandos e estagiários didáticos pós-graduandos.

Dentre outros desafios vividos por professores e estudantes, Crawford et al. (2020) enumeram a ausência ou a precariedade de infraestrutura de home office, com banda larga da Internet disponível, além da falta de domínio de recursos e competências digitais para projetar ou aprender no ensino digital. A garantia de uma infraestrutura de rede confiável é um dos elementos principais para o ERE, a fim de garantir participação nos momentos síncronos, usar recursos em tempo real, assistir a vídeos, baixar materiais, fazer upload, trabalhar com colegas em produções colaborativas (HUANG et al., 2020). Além disso, alguns cuidados também deveriam ser considerados para que os estudantes pudessem gerenciar de forma mais autônoma 
sua aprendizagem, sem contato presencial com colegas e equipe acadêmica (SANDARS et al., 2020). Em acréscimo, Meyer (2014) alerta que o estudante pode não dispor de tempo suficiente para as atividades, não ter experiência prévia com a aprendizagem online e não saber como agir, ter dificuldade em autorregular seu próprio comportamento, ser menos disposto a metodologia ativa ou preferir abordagens face a face.

A pesquisa americana Suddenly online (DIGITAL PROMISE; LANDER RESEARCH, 2020) captou a percepção de 1008 estudantes sobre o ensino e a aprendizagem remotos. Diferentes variáveis influenciaram o nível de satisfação dos alunos. Dentre oito estratégias de ensino online, as que geraram maior impacto na satisfação e com a aprendizagem foram: envio de mensagens pessoais aos estudantes (o que também favoreceu a motivação para o ensino remoto); apresentação de exemplos do mundo real; e as que incentivavam a autorreflexão do estudante sobre sua aprendizagem. Dentre os desafios para a realização do ensino remoto, Huang et al. (2020) reportam que vários estudantes não possuem as estratégias de estudo independente, processo autorregulatório e motivação, considerados fatores fundamentais para a aprendizagem online. Os autores ponderam que deve ser evitado o uso demasiado de diferentes aplicativos e plataformas, optando-se por recursos amigáveis e acessíveis. Para Sandars et al. (2020), o canal de comunicação e as orientações aos estudantes devem ser claros, acessíveis e bidirecionais, com mensagem explícita sobre as expectativas. Também indicam o uso dos recursos do próprio AVA para monitorar a realização das atividades pelos estudantes e identificar aqueles que precisam de orientação e apoio. A interatividade, a presença social e a presença cognitiva são recursos importantes para a motivação e o engajamento na aprendizagem online (Huang et al., 2020). Como há evidências empíricas da força preditiva da autorregulação da aprendizagem no sucesso em ambientes não lineares, a ação docente deve incluir a sua ativação e suporte aos estudantes (MOOS, 2018). Mas também deve haver medidas institucionais. Nessa direção, o Setor de Orientação Educacional do SAE convergiu suas ações para as especificidades do ensino remoto, por meio de atendimentos online; realização de lives, oficinas virtuais, webinários; produção de material de orientação aos estudantes e professores; e realização, em conjunto com a Faculdade de Educação, da disciplina eletiva Oficina de Autorregulação da Aprendizagem. Destacam-se os temas: como estudar quando não estou presencialmente na universidade; ingresso no ensino superior; estudo diário e para as avaliações; trabalho em grupo; ansiedade e regulação emocional; empregabilidade pós-pandemia.

Passados dois meses do início do semestre remoto, em um dia reservado no calendário acadêmico, foi realizada a avaliação de cursos com estudantes, professores e gestores acadêmicos. A maioria se apoiou em um questionário preparado e enviado previamente pela PRG, que buscava promover a identificação e a reflexão sobre o impacto da suspensão das atividades presenciais e possíveis encaminhamentos. Houve uma parceria importante entre as coordenações de curso e representações discentes na organização e na realização dessas reuniões. Os relatórios preparados tiveram seu conteúdo analisado, tendo como base o estudo da Universidad Nacional Autónoma de México (MENDIOLA et al., 2020), mostrando dificuldades enfrentadas pelos docentes nas dimensões logísticas (43,3\%), tecnológicas $(39,7 \%)$, pedagógicas $(35,2 \%)$ e socioafetivas $(14,9 \%)$, com resposta de $48,6 \%$ dos docentes daquela universidade. Essas dimensões foram usadas como categorias para a análise qualitativa dos relatos sobre a avaliação dos cursos frente a dois eixos: soluções/reconhecimento e preocupações/dificuldades. Nas dimensões Logística e Tecnológica, houve o reconhecimento do apoio da DEDH e da PRG e a constatação de aumento na taxa de acesso aos equipamentos e internet. Por outro lado, foram preocupações destacadas nas avaliações: o uso de diferentes AVAs nas disciplinas, exigindo domínio de diferentes plataformas; a participação em atividades síncronas; as condições técnicas para realizar a avaliação; as condições temporais, de espaço físico e disponibilidade de equipamentos para estudo e realização das atividades. 
$\mathrm{Na}$ dimensão pedagógica foram reconhecidos os esforços do corpo docente e das coordenações e o apoio da instituição para o ensino remoto; a grande e ativa participação dos estagiários e dos auxiliares nas atividades didáticas, estudantes de pós-graduação e graduação, respectivamente; o esforço e empenho dos estudantes; o valor da utilização de atividades assíncronas e síncronas, permitindo contato regular com os docentes responsáveis; e a importância do acolhimento, da compreensão e da flexibilidade por parte dos docentes no desenvolvimento do semestre. Como dificuldades, foram apontados aspectos relacionados à organização da disciplina (foco/objetivos, cronograma, critérios de avaliação e prazos, feedback, AVA), videoaulas longas, ausência de encontros síncronos ou encontros não disponíveis por meio de gravação, reduzida atividade em grupo, sobrecarga de atividades. Os estudantes relataram dificuldade em gerenciar os estudos e necessidade de investir maior tempo para a realização das atividades e para apreensão do conteúdo. Por outro lado, os docentes apontaram o impacto da baixa participação dos estudantes nos momentos síncronos, a obtenção de pouco feedback dos estudantes e reduzido domínio sobre o ensino remoto e os recursos digitais.

Interessante notar que, na pesquisa americana Time for Class (TYTON; DIGITAL PROMISE, 2020), com mais de 4.000 professores de mais de 1.500 instituições de ensino superior, $75 \%$ dos professores apontam como prioridade para o próximo semestre a busca e o uso de ferramentas digitais para promover o engajamento e a colaboração dos estudantes. Ao estudar a experiência da Universidade de Pequim com o ensino remoto, Bao (2020) identificou seis estratégias instrucionais para aprimorar a concentração e o engajamento dos estudantes: ter um plano alternativo diante de problemas inesperados; dividir o conteúdo em unidades de aprendizagem menores, organizado por módulos; tomar consciência da restrição da linguagem corporal e de expressões na comunicação com os estudantes e ênfase na voz; atuar em conjunto com assistentes de ensino que poderão oferecer suporte aos estudantes, mas também aos professores na incorporação e no uso de tecnologias; fortalecer a aprendizagem ativa e acompanhar a realização dos estudantes; combinar o ensino remoto e a autorregulação da aprendizagem nos momentos offline com feedback do professor. Essas preocupações também têm sido trazidas pelos professores da Unicamp, nos atendimentos online de suporte, nos fóruns da Comunidade de Prática e nas discussões dos webinários.

$\mathrm{Na}$ dimensão socioafetiva, foram evidenciadas ansiedade diante da pandemia, incertezas para finalização do semestre, preocupação com a formação (especialmente os concluintes), falta da convivência presencial, dificuldade de concentração e insegurança quanto às condições de retorno (estudantes e docentes). Diante disso, foram criados canais de acolhimento e de diálogo na comunidade, como a realização de rodas virtuais de conversa e apoio da coordenação de curso e professores; e atendimentos do SAE e do SAPPE, este último também com plantões presenciais. Toquero (2020), ao abordar o contexto específico da pandemia, aponta a necessidade de serviços médicos, de saúde mental e de cuidado ao estudante na modalidade online, visando ao acesso, à agilização e à adesão ao tratamento.

Com o objetivo de identificar como a comunidade da Unicamp (discentes, docentes, pesquisadores e funcionários) está vivenciando o desenvolvimento de suas atividades de forma remota, foi preparado pelo Observatório Institucional da Coordenadoria Geral da Universidade, com colaboração da PRG, um questionário padronizado que se encontra em fase final de aplicação. O estudo Time for Class (2020), por exemplo, explicita que a transição para o ensino remoto foi avaliada de modo positivo por $45 \%$ dos professores, para $38 \%$ deles foi indiferente e $17 \%$ referiram ser pior. Os resultados específicos sobre a percepção da comunidade Unicamp irão orientar a proposição e o desenvolvimento das novas ações da PRG em apoio aos docentes e estudantes.

Início de agosto de 2020, e a perspectiva de retorno para atividades presenciais continua incerta. Já se definiu que o segundo semestre letivo, a ser iniciado em meados de setembro de 
2020, também demandará um formato predominantemente remoto, apoiado em recursos digitais. Diante da gravidade da pandemia, o problema, que inicialmente exigia medidas emergenciais, agora impõe uma ação mais prolongada. Esse contexto de incertezas se reflete bem no artigo de Maloney e Kim (2020) sobre os cenários possíveis para o período letivo de 2020-2021, em 15 opções que compõem um contínuo entre o retorno integral das atividades presenciais e o ensino totalmente remoto. Diante da imprevisibilidade do momento, Toquero (2020) aconselha que os professores estejam preparados com uma versão remota de suas disciplinas, com alinhamento curricular a essa necessidade. Assim, para enfrentar esses cenários, novos programas de desenvolvimento profissional para a docência remota com uso de recursos digitais estão em preparação, enquanto as normativas vêm sendo atualizadas e ajustadas às necessidades de extensão para mais um semestre.

Também é relevante ponderar sobre o ponto trazido por Sandars et al. (2020) quanto ao impacto da experiência no ensino remoto para a composição de um ensino mais híbrido após a pandemia, que tire proveito das contribuições que os recursos digitais podem trazer, com oferta de conteúdos complementares, ou permitindo acesso a quem tem dificuldades ou àqueles que se adaptam melhor a modelos digitais de aprendizagem. Para isso, sugere-se o levantamento e a avaliação das experiências online, com renovação dos currículos, tornando-os mais sensíveis às necessidades de aprendizagem dos estudantes (TOQUERO, 2020).

A sistematização de boas práticas para o ensino remoto emergencial, organizada por Sandars et al. (2020), indica a adoção de um modelo de gerenciamento de mudanças e adesão a um AVA. Sobre a modalidade de aprendizagem online, sugestões de otimização do seu potencial incluem: momentos síncronos ao utilizar estratégias de sala de aula invertida, intercalar pequenas atividades de aprendizagem e disponibilizar a gravação no AVA; atividades em pequenos grupos virtuais, com objetivos claros e apoio às demandas dos estudantes; momentos assíncronos, alinhando as atividades com os objetivos de aprendizagem; vídeos-aula (gravados, de curadoria ou em tempo real) que promovam estudo pessoal, reflexão individual, discussão em grupo; uso de mídias sociais, dado seu longo alcance, priorizando o desenvolvimento das relações interpessoais, para comentar, oferecer feedback e emular o senso de pertencimento e noção de comunidade; e reflexão online individual ou em grupo. Por fim, as demais sugestões propõem oferecer conteúdo na forma digital, mesmo que de modo parcial, orientar adequadamente os estudantes; fomentar e dar suporte à cocriação de recursos e atividades online; e demonstrar o valor da mudança para a aprendizagem online e a abrangência no modo de estudar, atuar e se relacionar.

Embora os resultados acadêmicos no semestre só possam ser avaliados a partir de setembro de 2020, pode-se reconhecer que essa tem sido uma experiência profícua. Sabe-se, contudo, que os desafios atuais da pandemia ainda irão repercutir nos próximos anos (MALONEY; KIM, 2020). A crise de saúde se soma a uma crise econômica, social e cultural sem precedentes. Um relatório da Unesco (MARINONI; VAN'T LAND; JENSEN, 2020) mostrou que dois terços das instituições de ensino superior estavam fechadas em abril de 2020. Além de identificar os rápidos avanços observados em todo o mundo, chama atenção para o potencial impacto negativo esperado na adesão e na continuidade dos estudos por muitos estudantes do ensino superior, em razão da crise econômica. Mesmo para a situação particular da Unicamp, instituição pública e gratuita, as dificuldades do estudante e sua família podem comprometer a continuidade dos estudos. Isso exigirá soluções criativas para permitir a conclusão desse ciclo de formação.

No relatório da Unesco, salienta-se a agenda da igualdade e combate à iniquidade, do apoio necessário a estudantes, docentes, servidores e gestores acadêmicos diante de tantos desafios; a relevância das parcerias para permitir que as universidades possam continuar cumprindo sua missão de preparar as novas gerações de cidadãos e profissionais com qualidade 
e capacidade reflexiva, crítica e inovadora. Destacam-se as mudanças no processo de ensinoaprendizagem vivido como o ensino remoto emergencial, a oportunidade de repensar os cursos, as disciplinas, os currículos e os projetos pedagógicos. Aponta-se que a docência e a formação continuada para exercê-la passaram para o plano prioritário na agenda das instituições. Reconhece-se que a pandemia tem sido motivadora da reflexão experiencial sobre o papel de currículos híbridos, que combinem atividades presenciais e remotas, síncronas e assíncronas, com uso de recursos digitais, em propostas educacionais mais centradas na aprendizagem dos estudantes e flexíveis, que incluam capacidade de aprender ao longo da vida.

Mas também salienta-se a recuperação do papel da Universidade como suporte para promover apoio a governos e para assumir decisões sensíveis sobre as políticas públicas, além dos avanços específicos em pesquisa para responder aos novos desafios da pandemia e, mesmo, para oferecer serviços de saúde à população afetada. E destaca-se o estímulo a maior envolvimento dos docentes, discentes e servidores com a comunidade e sua contribuição, esclarecendo as informações relevantes sobre a própria pandemia e suas consequências e tornando a universidade uma grande parceira da sociedade. Esta percepção também se pode observar no Brasil e, em particular, em relação à contribuição das universidades públicas paulistas, e da Unicamp, em seu perfil ágil de resposta, na forma de pesquisa e desenvolvimento para atender a questões trazidas pela pandemia.

Sintetizando as lições aprendidas, temos: oportunidade de demonstrar que a universidade é uma instituição resiliente, ágil em suas respostas ao novo; reconhecimento da potência da relação empática entre estudantes e professores, acentuada pela condição da pandemia e da importância do ensino centrado no estudante, com equidade, flexibilidade e apoio institucional; premência da valorização das ações de desenvolvimento profissional continuado para a docência; potencial da aprendizagem com sala de aula invertida e trabalhos de grupo ou individuais remotos na solução de problemas ou desafios; possibilidade de utilizar estratégias educacionais híbridas que se beneficiem das melhores contribuições do ensino presencial e do remoto; reconhecimento do papel das avaliações formativas e processuais para a aprendizagem dos estudantes e como feedback sobre o ensino; relevância da manutenção de um canal aberto e ágil de comunicação entre a gestão central da universidade, gestores acadêmicos das unidades, docentes e estudantes; importância da troca de experiências com instituições nacionais e internacionais para ter modelos inspiradores.

No entanto, ainda é preciso ampliar os estudos sobre o impacto da Covid-19 no contexto educativo (BAO, 2020; TOQUERO, 2020), promover o monitoramento de dados institucionais e de sistemas, práticas baseadas em evidências e respostas às crises educacional, econômica e de emprego, ou mesmo, um novo surto (TOQUERO, 2020). Essas avaliações devem abranger as inovações de ensino e de currículo, mas também os serviços de apoio ao estudante e ações de desenvolvimento profissional docente. Crawford et al. (2020), além de corroborar a necessidade de investigações sobre abordagens pedagógicas diante da abrupta transição para o ensino remoto, anunciam a crucial ação colaborativa de todo o mundo quanto às melhores práticas no ensino remoto.

Finalmente, o novo semestre e o encerramento do ano letivo ainda serão enfrentados com muitas incertezas, incluindo quando será possível retomar as atividades presenciais e com que quantidade de pessoas nos campi. Atividades indispensáveis para estudantes que precisam concluir o curso não puderam ser realizadas de forma remota; está impossibilitada a experiência vivencial inigualável no campus universitário que os ingressantes não puderam ter; faltam o encontro e as trocas entre estudantes, professores, funcionários e tantas outras experiências formativas do cidadão, que se modificaram bruscamente neste ano tão singular. A recuperação parcial destes elementos essenciais da vida universitária deve nos motivar a sermos ainda mais criativos, enquanto o retorno presencial é um risco a muitas vidas. 


\section{Referências}

ARIAS, E.; ESCAMILlA, J.; LÓPEZ, A.; PEÑA, L. ¿Cómo perciben los docentes la preparación digital de la Educación Superior en América Latina? Junho, 2020. Disponível em: https://observatorio.tec.mx/edu-news/encuesta-preparacion-digital-docentes-universitariosamerica-latina. Acesso em: 01 jul. 2020.

BAO, W. Covid19 and online teaching in higher education. A case study of Peking University. Human Behavior and Emerging Technologies, v. 2, n. 2, p. 107-195, 2020. DOI: https://doi.org/10.1002/hbe2.191.

CRAWFORD, J.; BUTLER-HENDERSON, K.; JURGEN, R. MALKAWI, B. H.; GLOWATZ, M.; BURTON, R.; MAGNI, P.; LAM, S. Covid-19: 20 countries' higher education intra-period digital pedagogy responses. Journal of Applied Learning \& Teaching, v. 3, n. 1, 2020. DOI: https://doi.org/10.37074/jalt.2020.3.1.7.

DIAS-TRINDADE, S.; MOREIRA, J. A.; FERREIRA, A. G. Pedagogia(s) 2.0 em rede no ensino superior. In: DIAS-TRINDADE, S.; MOREIRA, J. A.; FERREIRA, A. G. (Coord.) Pedagogias digitais no Ensino Superior. Coimbra: Cinep.ipc, 2020. p. 9-23.

DIGITAL PROMISE; LANDER Research. Suddenly online: a national survey of undergraduates during the covid-19 pandemic, 2020. Disponível em: https://digitalpromise.org/wpcontent/uploads/2020/07/ELE_CoBrand_DP_FINAL_3.pdf. Acesso em: 27 jul. 2020.

FLORES, M. A.; GAGO, M. Teacher education in times of COVID-19 pandemic in Portugal: national, institutional and pedagogical responses. Journal of Education for Teaching, jul. 2020. DOI: $\quad$ https://doi.org/10.1080/02607476.2020.1799709. Disponível em: https://doi.org/10.1080/02607476.2020.1799709. Acesso em: 24 jul. 2020.

FRYER, L. K.; BOVEE, H. N. Supporting students' motivation for e-learning: Teachers matter on and offline. The Internet and Higher Education, n. 30, p. 21-29, 2016. DOI: https://doi.org/10.1016/j.iheduc.2016.03.003.

HERINGER, R. Democratização da educação superior no Brasil: das metas de inclusão ao sucesso acadêmico. Revista Brasileira de Orientação Profissional, v. 19, n. 1, p. 7-17, 2018. DOI: http://dx.doi.org/1026707/1984-7270/2019v19n1p7.

HODGES, C.; MOORE, S.; LOCKEE, B.; TRUST, T.; BOND, A. The difference between emergency remote teaching and online learning Friday. EDUCAUSE Review, March 27, 2020 Disponível em: https://er.educause.edu/articles/2020/3/the-difference-between-emergency-remoteteaching-and-online-learning. Acesso em: 24 jul. 2020.

HUANG, R. H. et al. Handbook on facilitating flexible learning during educational disruption: The Chinese experience in maintaining undisrupted learning in COVID-19 Outbreak. Beijing: Smart Learning Institute of Beijing Normal University, 2020. Disponível em: https://iite.unesco.org/wpcontent/uploads/2020/03/Handbook-on-Facilitating-Flexible-Learning-in-COVID-19-OutbreakSLIBNU_V2.0_20200324.pdf. Acesso em: 24 jul. 2020. 
MALONEY, E. J.; KIM, J. 15 cenários de outono: ensino superior em tempos de distanciamento social. Inside Higher Ed, Washington, D. C., abr. 2020. Disponível em: https://www.insidehighered.com/digital-learning/blogs/learning-innovation/15-fall-scenarios. Acesso em: 24 abr. 2020.

MARINONI, G.; VAN'T LAND, H.; JENSEN, T. The impact of COVID-19 on global higher education. International Higher Education, Special issue, p. 102, 2020. Disponível em: https://www.iau-aiu.net/IMG/pdf/iau_covid19_and_he_survey_report_final_may_2020.pdf.

Acesso em: 04 jul. 2020.

MENDIOLA, M. S.; HERNÁNDEZ, A. M. DEL P. M.; CARRASCO, R. T.; SERVÍN, M. DE A.; ROMO, A. K. H.; LARA, M. A. B.; VERGARA, C. A. J.; CAZALES, V. J. R. Retos educativos durante la pandemia de COVID-19: una encuesta a profesores de la UNAM. Revista Digital Universitaria, Ahead of print, 2020. Disponível em: https://www.revista.unam.mx/wpcontent/uploads/AOP.pdf. Acesso em: 12 maio 2020.

MEYER, K. A. (2014). Student engagement in online learning: What works and why, v. 40, n. 6, p. 1-114. Published online in Wiley Online Library (wileyonlinelibrary.com) DOI: https://doi.org/10.1002/aehe.20018.

MOOS, D. C. Emerging classroom technology: Using self-regulation principles as a guide for effective implementation. In: SCHUNK, D. H.; GREENE, J. A. Handbook of self-regulation of learning and performance. 2. ed. Taylor \& Francis, 2018. p. 243-253.

O'KEEFE, L.; RAFFERTY, J.; GUNDER, A.; VIGNARE, K. Delivering high-quality instruction online in response to COVID-19: Faculty playbook. Every Learner Everywhere, 2020. Disponível em: http://www.everylearnereverywhere.org/resources. Acesso em: 20 jun. 2020.

REDECKER, C. European framework for the digital competence of educators: DigCompEdu. PUNIE, Y. (Ed.). EUR 28775 EN. Publications Office of the European Union, Luxembourg, 2017, ISBN 978-92-79-73494-6, doi: https://doi.org/10.2760/159770, JRC107466.

SANDARS, J. et al. Twelve tips for rapidly migrating to online learning during the COVID-19 pandemic. MedEdPublish, v. 9, n. 1, p. 82, 2020. DOI: https://doi.org/10.15694/mep.2020.000082.1.

TOQUERO, C. M. Challenges and opportunities for higher education amid the Covid-19 pandemic: The Philippine context. Pedagogical Research, v. 5, n. 4, 2020. DOI: https://doi.org/10.29333/pr/7947.

TYTON; DIGITAL PROMISE. Time for class Covid-19 part 1: A national survey of faculty during Covid-19, 2020. Disponível em: https://tytonpartners.com/library/time-for-classcovid19-edition-part-1/. Acesso em: 27 jul. 2020.

\section{Sobre as autoras}

Eliana Amaral é graduada em Medicina (Faculdade de Medicina de Jundiaí), tem Mestrado, Doutorado e Livre-Docência em Tocoginecologia na Universidade Estadual de Campinas, onde é professora-titular de Obstetrícia. É Pró-Reitora de Graduação da Unicamp (2017-2021), 
Membro do Conselho Estadual de Educação do Estado de São Paulo, com mandato de agosto/2017 a agosto/2020. Realiza pesquisas nas áreas de HIV-AIDS e DST, infecções na mulher, saúde reprodutiva, qualidade de assistência obstétrica e ensino na saúde.

E-mail: elianaa@unicamp.br.

Soely A. J. Polydoro é graduada em Psicologia pela Pontifícia Universidade Católica de Campinas, tem Mestrado em Psicologia Escolar (Pontifícia Universidade Católica de Campinas) e Doutorado em Educação (Universidade Estadual de Campinas). É professora na Faculdade de Educação da Universidade Estadual de Campinas. É coordenadora geral do Espaço de Apoio ao Ensino e Aprendizagem (EA)2/Unicamp. Pesquisa: formação do estudante do ensino superior, processos de integração acadêmica, autoeficácia, autorregulação da aprendizagem e dimensões educativas associadas.

E-mail: polydoro@unicamp.br. 